\title{
Is case-specificity content-specificity? An analysis of data from extended-matching questions
}

\author{
Valerie Dory $\cdot$ Robert Gagnon $\cdot$ Bernard Charlin
}

\section{Erratum to: Adv in Health Sci Educ DOI 10.1007/s10459-009-9169-z}

Unfortunately, a mistake slipped through the proof reading process. The mistake is in the variance component numbers in Table 1 and the beginning of the results section. They should be divided by 100,000 . Below you will find the right table.

The online version of the original article can be found under doi:10.1007/s10459-009-9169-z..

V. Dory $(\bowtie)$

Centre Academique de Medecine Generale, Universite catholique de Louvain, Avenue Emmanuel, Mounier 53 (boite 5360), 1200 Brussels, Belgium e-mail: valerie.dory@uclouvain.be

R. Gagnon · B. Charlin

Centre de Pedagogie Appliquee aux Sciences de la Sante, Universite de Montreal, Montreal, Canada 
Table 1 Results of the generalisability G study

\begin{tabular}{|c|c|c|c|c|c|c|}
\hline & \multicolumn{2}{|l|}{2005} & \multicolumn{2}{|l|}{2006} & \multicolumn{2}{|l|}{ Mean } \\
\hline & $\begin{array}{l}\text { Variance } \\
\text { component }\end{array}$ & $\begin{array}{l}\% \text { of explained } \\
\text { variance }\end{array}$ & $\begin{array}{l}\text { Variance } \\
\text { component }\end{array}$ & $\begin{array}{l}\% \text { of explained } \\
\text { variance }\end{array}$ & $\begin{array}{l}\text { Variance } \\
\text { component }\end{array}$ & $\begin{array}{l}\% \text { of explained } \\
\text { variance }\end{array}$ \\
\hline $\mathrm{S}$ & 0.00645 & 2.64 & 0.00601 & 2.50 & 0.00623 & 2.57 \\
\hline $\mathrm{D}$ & 0.00397 & 1.63 & 0.00280 & 1.17 & 0.00339 & 1.40 \\
\hline T:D & 0.01969 & 8.07 & 0.01912 & 7.97 & 0.01941 & 8.02 \\
\hline I:T:D & 0.04206 & 17.23 & 0.04183 & 17.43 & 0.04195 & 17.33 \\
\hline S X D & 0.00159 & 0.65 & 0.00124 & 0.52 & 0.00142 & 0.59 \\
\hline S X T:D & 0.01168 & 4.78 & 0.01379 & 5.75 & 0.01274 & 5.27 \\
\hline S X I:T:D & 0.15867 & 65.00 & 0.15514 & 64.66 & 0.15691 & 64.83 \\
\hline Error domain & 39.75 & & 31.00 & & 35.38 & \\
\hline Error topic & 23.36 & & 27.58 & & 25.47 & \\
\hline Error item & 99.79 & & 97.57 & & 98.68 & \\
\hline
\end{tabular}

Relative G coefficients: 2005: 0.798; 2006: 0.794

Error domain: variance component for interaction between subject and domain/number of domains (4)

Error topic: variance component for interaction between subject and topic/number of topics (50)

Error item: variance component for interaction between subject and item/number of items (159)

$S$ subject, $D$ domain, $T: D$ topic nested in domain, I:T:D item nested in topic nested in domain, $S X D$ interaction between subject and domain, $S X T: D$ interaction between subject and topic nested in domain, $S X$ $I: T: D$ interaction between item nested in topic nested in domain 